\title{
Extracorporeal membrane oxygenation after pulmonary embolectomy - A case report
}

\author{
S. Kilwinger* and J.G. van der Hoeven \\ Department of Intensive Care, University Medical Centre Nijmegen, The Netherlands
}

\begin{abstract}
Massive pulmonary embolism (PE) is a life-threatening condition. Extracorporeal Life Support (ECLS) may be used for cardiopulmonary support, either as a bridge to surgical embolectomy or postoperative until cardiopulmonary recovery. We present a 35-year-old male suffering from massive PE who underwent surgical embolectomy and needed cardiopulmonary support for 6 days. Despite a complicated course, the patient recovered completely and was discharged home 36 days after admission. We review the existing literature regarding the use of ECLS in patients with massive PE.
\end{abstract}

\section{Introduction}

Massive pulmonary embolism (PE) is associated with a high mortality rate. In the International Cooperative Pulmonary Embolism Registry (ICOPER), 2454 patients with acute PE were registered to establish a 90 days all-cause mortality rate and identify factors associated with death [1]. One hundred and eight patients (4.5\%) had massive PE, defined as a systolic arterial pressure $<90 \mathrm{~mm} \mathrm{Hg}$ and 16 patients (15\%) with massive PE were first diagnosed at autopsy [2]. Overall mortality with massive pulmonary embolism was $52.4 \%$. The American Heart Association [3] defined massive PE as acute PE with sustained hypotension (systolic blood pressure $<90 \mathrm{~mm} \mathrm{Hg}$ for at least 15 minutes or requiring inotropic support, not due to other causes. Risk factors for mortality with acute PE are: age $>70$ years, cancer, clinical congestive heart failure, chronic obstructive pulmonary disease, systemic arterial hypotension (systolic blood pressure $<90 \mathrm{~mm}$ $\mathrm{Hg}$ ), tachypnea ( $>20$ breaths/min) and RV hypokinesis [1].

Treatment options for acute PE include systemic anticoagulation, intravenous thrombolytics, catheter-directed intrapulmonary thrombolytic infusion, catheter thrombectomy, rheolytic thrombectomy, and surgical Embolectomy [3-6].

As soon as massive PE is suspected, systemic anticoagulation (LMWH, UFH) should be given if there are no contra-indications. Systemic fibrinolysis is recommended for patients with massive acute $\mathrm{PE}$ and acceptable risk of bleeding complications. If fibrinolysis is contra-indicated or the patient remains unstable after fibrinolysis, either catheter embolectomy and fragmentation or surgical embolectomy is a reasonable alternative. Catheter embolectomy or surgical embolectomy may also be considered for patients with submassive acute PE who show clinical evidence of adverse prognosis [3].

\section{Case history}

A 35 -year-old male with no relevant medical history, presented to another hospital with progressive dyspnea for one month despite a short course of antibiotics and prednisone. His body mass index of 41 $\mathrm{kg} / \mathrm{m}^{2}$. On admission his vital signs were: blood pressure (BP) 109/72 $\mathrm{mmHg}$, heart rate (HR) $122 \mathrm{bpm}, \mathrm{SpO}_{2}$ of $89 \%$ without supplemental oxygen increasing towards $98 \%$ after administering 6 liters of oxygen. A CT pulmonary angiogram revealed extensive peripheral pulmonary emboli. Echocardiography showed a dilated right ventricle (Figure 1) and a huge thrombus in transit through the tricuspid valve (Figure 2). He was subsequently transferred to our hospital and immediately taken to the operating theatre. During anesthesia induction severe hypotension and desaturation developed. An immediate median sternotomy was performed. After opening the pericardium, a dilated and poorly contracting right ventricle was visible. Cardiopulmonary bypass was initiated, the right atrium was opened and a large thrombus $(15-20 \mathrm{~cm})$ was removed.

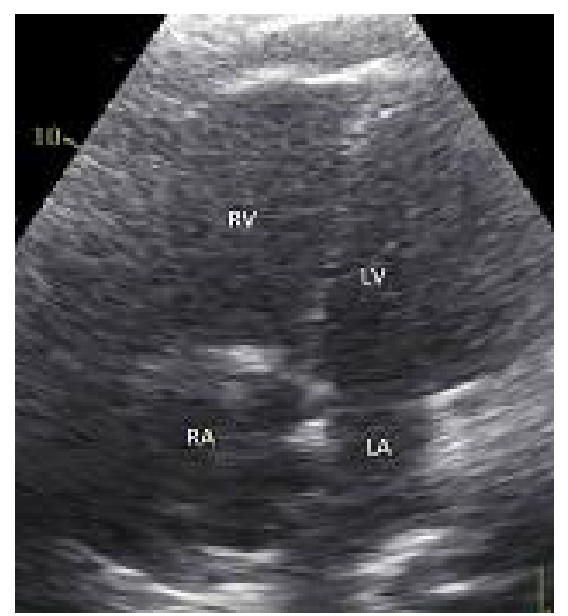

Figure 1. Transthoracic echocardiography - 4-chamber apical view showing an enlarged right ventricle and right atrium compatible with an acute increase in right ventricular afterload.

Correspondence to: S. Kilwinger, Department of Intensive Care, University Medical Centre Nijmegen, The Netherlands, E-mail: s.kilwinger@kpnplanet.nl

Key words: extracorporeal membrane oxygenation, massive pulmonary embolism

Received: June 18, 2015; Accepted: July 06, 2015; Published: July 10, 2015 


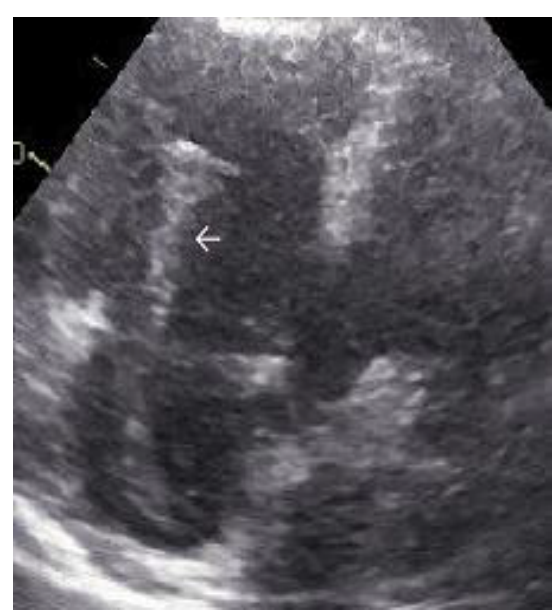

Figure 2. Transthoracic echocardiography showing right atrium and right ventricle with a large in-transit thrombus (white arrow) crossing the tricuspid valve.

Subsequently, the pulmonary trunk was opened. A similar but smaller $(10 \mathrm{~cm})$ thrombus was removed from the bifurcation. More peripheral organized thrombi were removed from the left and right pulmonary artery. Weaning from cardiopulmonary bypass was initially successful with norepinephrine, dobutamine and milrinone despite severe right ventricular dilatation. The patient was decannulated but during closure of the sternum, the patient became progressively unstable resulting in asystole. Internal cardiac massage was performed for 7-8 minutes and a central venous-arterial (V-A) ECLS system (CARDIOHELP, Maquet) was installed with a flow of 4-5 liters per minute. The patient was then transferred to the ICU. Despite several reexplorations for extensive bleeding and a period of recurrent ventricular fibrillation with a normal coronary angiogram, there was a slow improvement of the patient's right and left ventricular function. After 6 days of ECMO, the patient was successfully weaned and decannulated. Mediastinitis with B. cereus was treated with ciprofloxacin. Vacuum assisted closure therapy was started. Twelve days after decannulation the patient was extubated. The sternum was subsequently closed using a Sternalock system. Thirty-six days after admission he was discharged home without neurological impairment. Echocardiography still showed an moderate to severe hypokinetic right ventricle. Five months after discharge he leads a completely independent life.

\section{Discussion}

We describe a patient with massive pulmonary embolism who underwent a surgical embolectomy complicated by severe right- and left ventricular failure. Despite prolonged ECLS support and a complicated ICU course, the patient survived without important residual defects.

The last two decades several case series and case reposts were published describing patients with acute PE and ECLS. We could not find any randomized controlled trials.

Most patients had peripheral cannulation using the femoral vein and artery. Other locations used were the subclavian artery, axillary artery or carotid artery and internal jugular vein. If ECLS is started at the end of a surgical embolectomy, central cannulation is a viable option.

ECLS in acute PE can be used to stabilize the patient and serve as a bridge to definite treatment $[7,8]$. Sometimes the patient will recover without definite treatment [9]. It can also be used to transport the patient to another hospital [10].
Malekan et al. [9] described a series of 29 patients with (sub) massive PE treated between 2005 and 2011. Twenty-six patients underwent surgical embolectomy. In four patients (average age 46.8 years, range 23 to 73 years) the estimated risk for surgery was too high and these four patients were placed on ECLS. All patients needed regular red blood cell transfusions (average 5.25 units) for oozing at the arterial cannulation site. ECLS was successfully weaned in 3 patients after 5 or 6 days. The last patient did not improve after 10 days of ECLS and underwent rescue surgery. All 4 patients survived. Munakata et al. [11] examined 35 patients with massive PE between 1992 and 2008 and 10 patients were placed on ECMO. Thirty day mortality rate was 30\%. Maggio et al. [12] described 21 patients with acute PE and ECLS between 1992 and 2005. Thirteen patients (62\%) survived. They reported several complications: hemodynamic instability (57\%), cannulation site bleeding (38\%), cardiac arrhythmias (38\%), intracerebral hemorrhage or infarction (24\%), surgical site bleeding (24\%), infection (24\%), creatinine $>3.0$ $\mathrm{mg} / \mathrm{dL}$ (24\%), hyperbilirubinemia (19\%), pneumothorax (14\%), oxygenator failure (14\%), heparin-induced thrombocytopenia (HIT) in one patient, soft tissue infection of the femoral cannulation site (one patient), femoral artery pseudo-aneurysm (one patient), dislodged cannula (one patient) and multi-organ failure in one patient.

Yusuff et al. [13] reviewed case reports and case series published in the last 20 years. They included 19 papers (including the three above mentioned case series) describing a total of 78 patients. Overall survival rate was $70,5 \%$. Definite treatment was either surgical embolectomy, catheter embolectomy, thrombolysis, a combination of these, or none (ECLS alone). They did not find a relationship between definite treatment and mortality.

We conclude that V-A ECMO has demonstrated effectiveness in providing cardiopulmonary support for the patient with a massive PE (either too unstable for fibrinolysis or surgery, or after treatment) [5,7-13]. Peripheral cannulation (femoral vessels) is a simple technique and will usually suffice. If conservative therapy is unable to stabilize hemodynamics, the patient should be transferred to a center with experience in extracorporeal life support techniques.

\section{References}

1. Goldhaber SZ, Visani L, De Rosa M (1999) Acute pulmonary embolism: clinica outcomes in the International Cooperative Pulmonary Embolism Registry (ICOPER) Lancet 353: 1386-1389. [Crossref]

2. Kucher N, Rossi E, De Rosa M, Goldhaber SZ (2006) Massive pulmonary embolism. Circulation 113: 577-582. [Crossref]

3. Jaff MR, McMurty MS, Archer SL, Cushman M, Goldenberg N, et al. (2011) Management of massive and submassive pulmonary embolism, iliofemoral deep vein thrombosis, and chronic thromboembolic pulmonary hypertension. A scientific statement from the American Heart Association. Circulation 123: 1788-1830. [Crossref]

4. Brahmbhatt T, Tutton S, Mannebach P, Cinquegrani M (2007) Rheolytic thrombectomy in patient with massive pulmonary embolism: a case report and review of literature. Catheter Cardiovasc Interv 70: 802-806. [Crossref]

5. Griffith KE, Jenkins E, Haft J (2009) Treatment of massive pulmonary embolism utilizing a multidisciplinary approach: a case study. Perfusion 24: 169-172. [Crossref]

6. Kucher N, Goldhaber SZ (2005) Management of massive pulmonary embolism. Circulation 112: e28-32. [Crossref]

7. Deehring R, Kiss AB, Garrett A, Hillier AG (2006) Extracorporeal membrane oxygenation as a bridge to surgical embolectomy in acute fulminant pulmonary embolism. Am J Emerg Med 24: 879-880. [Crossref]

8. Kawahito K, Murata S, Adachi H, Ino T, Fuse K (2000) Resuscitation and circulatory support using extracorporeal membrane oxygenation for fulminant pulmonary embolism. Artif Organs 24: 427-430. [Crossref] 
9. Malekan R, Saunders PC, Yu CJ, Brown KA, Gass AL, et al. (2012) Peripheral extracorporeal membrane oxygenation: comprehensive therapy for high-risk massive pulmonary embolism. Ann Thorac Surg 94: 104-108. [Crossref]

10. Hori D, Tanaka M, Kohinata T, Kimura C, Naito K, et al. (2010) Successful usage of extracorporeal membrane oxygenation as a bridge therapy for acute pulmonary embolism between hospitals. Gen Thorac Cardiovasc Surg 58: 283-286. [Crossref]
11. Munakata R, Yamamoto T, Hosokawa Y, Tokita Y, Akutsu K, et al. (2012) Massive pulmonary embolism requiring extracorporeal life support treated with catheter-based interventions. Int Heart J 53: 370-374. [Crossref]

12. Maggio P, Hemmila M, Haft J, Bartlett R (2007) Extracorporeal life support for massive pulmonary embolism. $J$ Trauma 62: 570-576. [Crossref]

13. Yusuff HO, Zochios V, Vuylsteke A (2015) Extracorporeal membrane oxygenation in acute massive pulmonary embolism: a systematic review. Perfusion [Epub ahead of print].

Copyright: $@ 2015$ Kilwinger S. This is an open-access article distributed under the terms of the Creative Commons Attribution License, which permits unrestricted use, distribution, and reproduction in any medium, provided the original author and source are credited. 